\title{
Palliative pelvic radiotherapy for gynaecologic cancer
}

\author{
Julia Skliarenko • Elizabeth A. Barnes
}

Received: 13 June 2012 / Accepted: 21 June 2012 / Published online: 18 July 2012

(C) Springer-Verlag 2012

\begin{abstract}
Background Radiotherapy delivered with palliative intent can relieve many of the distressing pelvic symptoms associated with advanced, recurrent, or metastatic gynaecologic cancer.

Results Series in the literature mainly focus on the outcomes of bleeding and pain for which high response rates are seen. This article reviews the hypofractionated palliative radiotherapy regimes and outcomes reported in the literature.

Conclusion Treatment should be individualized for each patient, taking into account symptom burden and etiology, risk of toxicity, performance status and life expectancy.
\end{abstract}

Keywords Gynaecologic cancer · Palliation · Recurrent . Radiotherapy $\cdot$ Bleeding $\cdot$ Pain

\section{Introduction}

In the United States, it is estimated that there will be 88,750 new gynaecological cancer cases (endometrium, ovary, cervix, vulva, vagina) and 29,520 gynaecologic cancer deaths in 2012 [1]. The death rate from ovarian cancer has decreased by $1.9 \%$ per year from 2004 to 2008, and has remained stable for endometrial cancer. Mortality rates for cervix cancer declined rapidly in the past decades as a result of screening, but have slowed in recent years. Patients with locally advanced, metastatic and recurrent gynaecological malignancies can experience a broad spectrum of physical and psychological symptoms requiring medical attention

J. Skliarenko $\cdot$ E. A. Barnes $(\bowtie)$

Odette Cancer Centre,

2075 Bayview Avenue,

Toronto, Ontario, Canada M4N 3M5

e-mail: toni.barnes@sunnybrook.ca and management [2]. Vaginal bleeding and pain, together with bowel, bladder, and lymphovascular obstruction, may all be caused by progressive abdominopelvic disease. These symptoms as well as side effects from previous treatments impact patients' quality of life as well as affect their psychological well-being. Distant metastases to bone, brain, liver, and lung can also occur. Local radiotherapy (RT) can be used to achieve symptom palliation and improve quality of life. Care for these patients is often optimized via a multidisciplinary approach involving gynaecological, medical, and radiation oncologists, as well as palliative care physicians, family physicians, psychology, nurses, social workers, dieticians, and spiritual care workers. Each member of the team has a unique and valuable role and their involvement should be considered at various stages of patient care. This paper focuses on the role of palliative RT in management of pelvic symptomscaused by incurable gynaecologic malignancies.

\section{Palliative care and palliative radiotherapy}

A World Health Organization statement describes palliative care as "an approach that improves the quality of life of patients and their families facing the problems associated with life-threatening illness, through the prevention and relief of suffering by means of early identification and impeccable assessment and treatment of pain and other problems, physical, psychosocial and spiritual" [3]. This is appropriate at all stages of a patient's cancer care; however, when the disease is incurable and life expectancy limited, the goals of care shift focus on symptom control and quality of life.

RT plays an important role in palliative management of advanced gynaecological malignancies, particularly with regard to haemostasis and pain relief. The goals of palliative 
RT are to provide durable and timely symptom relief while minimizing side effects, resource utilization and the number of visits to the cancer centre [4]. Secondary aims include tumour regression and short recovery time. Palliative RT may also prophylactically address an area with a high likelihood of causing symptoms in the future. As tumours do not need to be completely eradicated to achieve symptom relief, typically a short course of treatment delivering a high dose per fraction is recommended in the palliative setting [5]. Dose per fraction is related to late toxicity but many patients will not live long enough to be at risk [6]. However, care needs to be taken especially when considering retreating the same site as physicians are generally poor at survival estimation [7].

A number of hypofractionated fractionation schemes have been described for palliation ofgynaecological malignancies [2]. They vary from $30 \mathrm{~Gy}$ in ten fractions, to shorter courses of 3.7 Gy delivered twice daily in four fractions, to a single 8-Gy dose in one fraction. Many of these fractionation regimens canbe repeated. Decision regarding radiation fractionation regimen is based on a number of different factors including: tumour characteristics, imaging results, previous radiation treatment and response to said treatment, patient's performance status and life expectancy, and current or future systemic therapy. Physician preference and training also play a role in decision making. Administration of palliative RT for gynaecologic cancer may be delivered using the external beam approach as well as brachytherapy.

\section{Site-specific symptoms}

\section{Cervical cancer}

Worldwide, cervical cancer is the third most common cancer affecting women with 530,000 new cases seen in 2008 [8]. More than $85 \%$ of cases are seen in developing countries. Cervix cancer is both preventable with the HPV vaccine and screening [9], and curable when detected early, but in countries where access to medical care is not readily available the majority of patients present with advanced disease. RT, either radical or palliative in intent, is the mainstay of treatment [10]. Approximately 12,170 new cases of cervical cancer and 4,220 deaths from the disease are estimated for 2012 in the United States [1]. Patients with locally advanced disease can present with vaginal bleeding and discharge, pelvic pain, renal failure, obstructive symptoms, fistulas (cervicovesical, vesicovaginal, cervicorectal, rectovaginal), and lymphedema of the lower extremities. Pelvic and paraaortic adenopathy can cause pain due to bony destruction, nerve root impingement, and invasion into surrounding musculature.

\section{Endometrial cancer}

In North America, endometrial cancer is the fourth most common cancer that affects women [1]. In United States, 47,130 new cases and 8,010 deaths due to endometrial cancer are expected in 2012 [1]. The majority of patients $(70 \%)$ are diagnosed with early stage disease [11], presenting with postmenopausal bleeding and undergo definitive surgery with adjuvant radiation and/or chemotherapy treatment tailored to the surgical pathology. Patients with locally advanced or recurrent disease can present with additional symptoms such as pain secondary to bulk of primary disease or regional adenopathy, pain due to erosion into pelvic bony structures and musculature, and obstructive symptoms due to tumour mass or peritoneal seeding. In addition patients presenting with early stage disease are occasionally not amenable to curative treatment with surgery or radical RT due to medical comorbidities.

\section{Ovarian cancer}

Ovarian cancer is the leading cause of gynaecologic cancer death in North American women [11]. An estimated 22,280 new cases of ovarian cancer and 15,500 deaths are expected in the United States in 2012 [1]. Early stage is disease is asymptomatic, and therefore the majority of patients present with advanced-stage disease. Symptoms at presentation can include abdominal bloating and pain, early satiety, vague but persistent gastrointestinal complaints, urinary frequency or urgency, change in bowel habits, shortness of breath, as well as obstructive symptoms, such as nausea, vomiting, and constipation or obstipation. Platinumbased chemotherapy is the mainstay of treatment, but once platinum-insensitive the response rates to chemotherapy fall to $10-15 \%$ [12]. Local palliative RT can produce response rates of 50-80\%, and is an underutilized effective treatment modality for symptoms due to local mass effect.

\section{Vaginal cancer}

Vaginal cancer is rare, comprises $1-2 \%$ of all gynaecological malignancies [11]. In 2012, an estimated 2,680 women in the United States will be diagnosed with vaginal cancer, while estimated 840 will die from this disease [1]. Patients with advanced disease may present with vaginal bleeding and discharge; pelvic pain, dyspareunia, urinary symptoms, tenesmus, defecation problems can result from extension into the urethra, bladder, anus, and rectum.

\section{Vulvar cancer}

Vulvar cancer is relatively rare and represents approximately $4 \%$ of all gynaecologic malignancies. A total of 4,490 
new cases and 950 deaths are estimated for 2012 in the United States [1]. Vulvar cancer is most commonly seen in elderly women and associated with lichen sclerosis, although HPV-related disease can be seen in a younger population [13]. Neglect in the elderly population can lead to patients not presenting until their disease has progressed to the point of causing distressing symptoms. Curative treatment may not be possible due to the advanced stage of disease or poor patient performance status. Symptoms from advanced vulvar disease can include pain and pruritis, malodourous discharge, bleeding, and obstructive bladder and bowel symptoms [14]. Regional spread can result in fixed, ulcerated inguinal nodes, leading to skin ulceration with and bleeding and discharge, lymphedema and deep vein thrombosis, and patients may exsanguinate due to erosion into the femoral artery. Local skin metastases can also occur over the groin and perineum leading to pain, bleeding and ulceration. A short course of RT can produce dramatic regression of disease and symptom relief.

\section{Palliative radiotherapy for symptom management}

\section{Vaginal bleeding}

Vaginal bleeding is one of the most common and most distressing symptoms of recurrent or advanced gynaecologic malignancy, and is the most frequent reason for and outcome reported in trials examining palliative pelvic RT. Patients with cervix cancer may present with heavy vaginal bleeding requiring transfusion and intravascular volume replacement. Vaginal packing with hospital admission with urgent initiation of RT may be indicated. Care must be taken to ensure that patients with potentially curable disease are not given multiple high dose per fraction treatments in an attempt to control haemostasis which would later preclude delivering of a radical course of RT.

Hypofractionated RT provides effective and efficient palliation of vaginal bleeding in gynaecologic cancer. Starting with Boulware et al.'s [15] report in 1979, the MD Anderson experience of three monthly fractions of 10 Gy to the whole pelvis, other centres have subsequently reported their experience using this dose/fraction. The specific indications for repeating the second and third fractions are variable and not always reported, but generally include having a response to treatment and remaining in satisfactory performance status. Boulware et al. reported on 86 patients; 58 patients went on to receive a second and 20 patients received a third 10-Gy fraction. Pelvic bleeding was controlled in $45 \%$ of cases after the first, $85 \%$ after the second, and $100 \%$ after three fractions. Hodson and Krepart [16] delivered 10 Gy for three fractions to 27 patients; 24 of these patients received all three fractions, with $100 \%$ alleviation in bleeding [16].
Halle et al. [17] treated 42 patients; for those receiving a third fraction, the dose was reduced from 10 to $7 \mathrm{~Gy}$. Onsrud et al. [18] reported on 64 patients using monthly 10-Gy fractions, with the majority of patients receiving two fractions. Bleeding cessation was seen in $90 \%$ of patients. Finally, Mishra et al. [19] reported on 100 patients in India; in their report, 61 patients received the second and 33 the third fraction. The overall response rate for bleeding was $100 \%$ [19] (Table 1).

Overall late toxicity rate was $6-12 \%$ in these series, occurring 9-10 months after treatment $[17,18]$. It is difficult to assess the true rate of late complications from these 10Gy fractions. All series were retrospective, not all reported the complication rate, follow-up was incomplete, survival limited and "complications" such as fistula formation can be difficult to distinguish from disease progression. In addition, patients were mainly treated with large APPA fields $(15 \times$ $15 \mathrm{~cm}$ whole pelvis) and low energy photons. Today, using high energy photons and CT-based planning, the dose distribution can be more homogeneous and the dose and volume of bowel treated reduced. This may translate into lower rates of both acute and late treatment toxicity. Late complications were typically seen after 9 months, which led several authors to conclude that this treatment regime should be limited to patients with life expectancy less than 9 or 12 months [17, 18]. Alternately, as a correlation between symptom response and number of fractions was not always seen, the third fraction could be omitted to reduce the likelihood of late toxicity [20]. Survival can be difficult to accurately predict however; for example, Onsrud et al. [18] found that $28 \%$ of patients with an expected survival of $<12$ months actually lived longer than this.

Building on these single-institution experiences, the RTOG 7905 trial utilized this $10 \mathrm{~Gy} \times 3$ regime concurrently with misonidazole (a hypoxic cell sensitizer), in patients with various advanced pelvic malignancies [21]. There was a very high rate ( $45 \%)$ of late GI complications which lead to the trial being prematurely closed. This led to the RTOG prospectively investigating the use of a lower dose per fraction to reduce the incidence of late toxicity [22]. Two days of 3.7 Gy (BID) dose repeated monthly up to three

Table 1 Palliative pelvic radiotherapy for symptomatic advanced gynaecologic cancers

\begin{tabular}{lc}
\hline Symptom & Overall response rate \\
\hline Bleeding & $45-100 \%[15-19,26-28]$ \\
Pain & $0-83 \%[15-19,23]$ \\
Discharge & $39-49 \%[18,19]$ \\
Obstruction $^{\mathrm{a}}$ & $19-100 \%[15,23,29-31]$ \\
\hline
\end{tabular}

${ }^{a}$ Definition is often vague, and can include hydronephrosis, tenesmus, obstipation, rectosigmoid, gastric outlet or obstruction at stomal site 
times (total dose of 14.8 Gy per cycle, total overall dose of $44.4 \mathrm{~Gy}$ ) was used. Only one grade 3 late GI toxicity was reported in 142 evaluable patients; therefore, this was moved to a phase 3 trial (RTOG 8502) randomizing between 2- and 4-week breaks between cycles [23]. A total of 153 patients were accrued, 73 of whom were experiencing vaginal bleeding. No significant overall difference in symptom control was found withvaginal bleeding controlled in $97 \%$ of patients. The crude late complication rate was $6 \%$ with no significant difference seen between the 2- and 4-week arms [24]. This was a major reduction in toxicity from the previous RTOG trial, but the BID regimen required a significant time commitment from patients and those from out of town often required hospital admission. A later analysis limited to cervix cancer patients confirmed the findings in the large pelvic malignancy group - similar symptom response rates but lower complications using the RTOG 8502 regimes [25].

The PMH group published their 10-year retrospective experience using 0-7-21 (typically 7 Gy per fraction days $0,7,21)$ in the advanced gynaecology population [26]. Fifty-one patients were identified, of whom 48 received at least two fractions, and the response rate to vaginal bleeding was $92 \%$. Severe late toxicity was reported in two patients (sacral insufficiency fracture and death after surgery for bowel obstruction). This schedule is comparable to other hypofractionated regimes with regard to efficacy and toxicity. The authors report that one of the advantages of $0-7-21$ is the short treatment intervals, which allowed the patients to be assessed for progression and acute toxicity, and reassessed on day 21 as to whether to receive the last fraction.

Prolonged fractionation schemes have been reported in patients with ovarian cancer. This population has typically been heavily pretreated with chemotherapy, and may have a longer expected life expectancy. Corn et al. [27] reported on 33 patients treated mainly in the pelvis with a response rate for bleeding of $90 \%$. They reported that patients who received a biologically effective dose of at least $44 \mathrm{~Gy}_{10}$, equal to 35 Gy in 14 fractions, had a higher chance of achieving a complete response. Choan et al. [12] reported their experience with 53 patients, 25 were complaining of vaginal bleeding and their response rate to treatment was $100 \%$, with $88 \%$ complete response. In this study, 30 Gy in ten fractions and $20 \mathrm{~Gy}$ in five fractions were the most commonly used regimes [12].

In clinical practice, commonly used regimes for palliation include $30 \mathrm{~Gy}$ in ten fractions, 20 Gy in five fractions or single fractions of 5-10 Gy. The frequency that these regimes are used has not been reported in the literature. Whether there is any benefit in symptom control with dose escalation for gynaecologic malignancies has not been examined prospectively.
In summary, RT is a very effective and efficient method to provide a complete or partial, durable response for vaginal bleeding. A number of different fractionation schemes have been shown to be effective, with acceptable rates of acute and late toxicity.

\section{Pelvic pain}

Pelvic pain is a common symptom complaint in patients with advanced gynaecological malignancy [2]. Pain can result from the primary tumour, adenopathy, metastases entrapping nerve roots and trunks within the pelvis, or tumour extension to pelvic bone or musculature. A number of studies have shown RT to be an effective means of pelvic pain alleviation which may be secondary to reduction in tumour size, or mechanisms similar to that seen with pain relief from bone metastases for lesions invading bone.

While bleeding has been the outcome most often reported for palliative RT in gynaecologic cancer, alleviation of pelvic pain has also been described. There is difficulty in interpreting results as validated instruments were not used, and opioid and coanalgesic use was not taken into account. The duration of pain relief was also difficult to assess with the retrospective nature of the studies and patients being lost to follow-up. Boulware et al. [15] found complete or partial pain relief in $45 \%$ of patients after a single $10 \mathrm{~Gy}, 59 \%$ improvement after two fractions, and $63 \%$ improvement after three fractions. Hodson and Krepart [16] reported pain relief in six patients, but the number of patients with pain at baseline was not given. Halle et al. [17] reported complete pain relief in 2/9, and partial relief in $2 / 9$ patients, and noted that symptom relief was not correlated with number of fractions given. Mishra et al. [19] reported pain relief in 23 out of 48 patients. Results of the phase III RTOG 8502 trial reported pain relief in $68 \%$ of patients [23]. Corn et al. [27], who treated 33 patients with refractory ovarian cancer to a total dose of 44 Gy in 2.5-Gy fractions, reported pain relief in $83 \%$ of patients. Onsrud et al. [18], however, found no pain relief among six patients with pain at baseline.

\section{Vaginal discharge}

Malodourous vaginal discharge from cervix or endometrial cancer is a debilitating symptom for patients often isolating them from social contacts. Onsrud et al. [18] reported $39 \%$ (7/18) symptom relief with repeated 10-Gy fractions. Mishra et al. [19] reported 69 cervix cancer patients at presentation complained of vaginal discharge, and $49 \%$ overall relief was seen with no incremental gain from the third 10-Gy fraction. Patients with advanced vulvar primaries can also present with malodorous discharge. Reports on palliative RT outcomes or the symptomatic response to radical treatment are lacking in the literature. 
Impressive rates of regression are seen with preoperative chemoradiation for advanced vulvar disease, 4,760 cGy with concurrent $5-\mathrm{FU}$ resulted in $46.5 \%$ of patients having no visible vulvar disease at the time of planned surgery [28].

\section{Obstruction}

Large bowel obstructive symptoms can be seen in patients with locally advanced or recurrent disease in the pelvis, and defunctioning colostomy may be required. Hydronephrosis (with resultant renal dysfunction if bilateral) can result from compression of the ureters along their course by enlarged para-aortic or pelvic nodes, soft tissue deposits or primary pelvic disease extending to pelvic sidewall. Nephrostomy tubes may be required to for patients presenting in acute renal failure. When there is an identifiable mass causing the obstruction, local RT can be given with the goal of shrinking tumour and preventing or relieving symptoms.

Boulware et al. [15] reported relief of obstructive uropathy (as demonstrated by intravenous pyleography), with improvement in edema/uremia as the reported outcome in $24 \%, 75 \%$, and $75 \%$ of patients receiving 1, 2, and 3 fractions of pelvic RT, respectively. This correlated with increasing rates of objective tumour response, suggesting a correlation with tumour shrinkage. RTOG 8502 reported $88 \%(14 / 16)$ relief of tenesmus or obstipation; however, it is not clear whether these patients had gastrointestinal or gynaecologic primaries [23].

In the ovarian cancer population, May et al. [29] and Tinger et al. [30] report a $63 \%$ and $75 \%$ response for obstruction of gastrointestinal and genitourinary tract. May reported that these obstructions were easy to locate, involving the rectosigmoid, gastric outlet or stomal site. Small bowel obstruction can be seen with peritoneal metastases from ovarian cancer, but is usually multifocal and multifactorial in etiology. There is rarely a single causative tumour deposit that can be identified, and so the role or palliative RT in this situation is limited. Lymphedema can be secondary to pelvic disease, but again is often multifactorial in etiology. Deep vein thrombosis and late complications from surgery and RT should be considered in the differential for lower extremity edema. Choan et al. reported $5 / 5$ patients had a partial response to RT [12], and other research groups all reported minimal response after RT [28, 29].

\section{Summary}

RT can provide effective palliation for pelvic symptoms secondary to advanced, metastatic or recurrent gynaecologic cancer. The highest response rates are seen for bleeding and pain, but obstruction and vaginal discharge can also respond to treatment. Various efficacious dose fractionation regimes have been reported in the literature. Treatment should be individualized for each patient, taking into account symptom burden and etiology, risk of toxicity, performance status and life expectancy.

\section{References}

1. Siegel R, Naishadham D, Jemal A (2012) Cancer statistics, 2012. CA Cancer J Clin 62(1):10-29

2. Smith SC, Koh WJ (2001) Palliative RT for gynaecological malignancies. Best Pract Res Clin Obstet Gynaecol 2:265-278

3. http://www.who.int/cancer/palliative/definition/en/. Accessed 11 June 2012

4. Chow E, Wong R, Hruby G et al (2001) Prospective patient-based assessment of effectiveness of palliative RT for bone metastases in an outpatient radiotherapy clinic. Radiother Oncol 61:77-82

5. Kirkbride P, Barton R (1999) Palliative radiation therapy. J Palliat Med 2(1):87-97

6. Spiro S, Douse J, Read C et al (2008) Complications of lung cancer treatment. Semin Respir Crit Care Med 29:302-318

7. Chow E, Davis L, Panzarella T et al (2005) Accuracy of survival prediction by palliative radiation oncologists. Int $\mathrm{J}$ Radiat Oncol Biol Phys 61(3):870-873

8. Ferlay J, Shin HR, Bray F et al (2010) GLOBOCAN 2008 v1.2, Cancer incidence and mortality worldwide: IARC Cancer Base No. 10. International Agency for Research on Cancer, Lyon, France. Available from: http://globocan.iarc.fr. Accessed on 11 June 2012

9. Garland SM, Hernandez-Avila M, Wheeler CM et al (2007) Quadrivalent vaccine against human papillomavirus to prevent anogenital diseases. N Engl J Med 356(19):1928-1943

10. van Lonkhuijzen L, Thomas G (2011) Palliative radiotherapy for cervical carcinoma, asystematic review. Radiother Oncol 98 (3):287-291

11. SEER Stat Fact Sheets: Corpus and Uterus, NOS. http://seer.cancer.gov/ statfacts/html/corp.html. Accessed on 11 June 2012

12. Choan E, Quon M, Gallant V et al (2006) Effective palliative radiotherapy for symptomatic recurrence or residual ovarian cancer. Gynecol Oncol 102:204-209

13. Ueda Y, Enomoto T, Kimura T et al (2011) Two distinct pathways to development of squamous cell carcinoma of the vulva. J Skin Cancer 951250:1-7

14. Barnes EA, Thomas G (2006) Integrating radiation into the management of vulvar cancer. Semin Radiat Oncol 16(3):168-176

15. Boulware RJ, Caderao JB, Delclos L et al (1979) Whole pelvis megavoltage irradiation with single doses of $1000 \mathrm{rad}$ to palliate advanced gynecologic cancers. Int J Radiat Oncol Biol Phys 5:333-338

16. Hodson DI, Krepart GV (1983) Once-monthly radiotherapy for the palliation of pelvic gynecological malignancy. Gynecol Oncol $16: 112-116$

17. Halle JS, Rosenman JG, Varia MA et al (1986) 1000 cGy single dose palliation for advanced carcinoma of the cervix or endometrium. Int J Radiat Oncol Biol Phys 12:1947-1950

18. Onsrud M, Hagen B, Strickert T (2001) 10-Gy single-fraction pelvic irradiation for palliation and life prolongation in patients with cancer of the cervix and corpus uteri. Gynecol Oncol 82:167171

19. Mishra SK, Laskar S, Muckaden MA et al (2005) Monthly palliative pelvic radiotherapy in advanced carcinoma of uterine cervix. J Can Res Ther 1(4):208-212 
20. Adelson MD, Wharton JT, Delclos L et al (1987) Palliativeradiotherapy for ovarian cancer. Int J Radiat Oncol Biol Phys 13(1):17-21

21. Spanos WJ Jr, Wasserman T, Meoz R et al (1987) Palliation ofadvanced pelvic malignant disease with large fraction pelvic radiation andmisonidazole: final report of RTOG phase I/II study. Int J Radiat Oncol Biol Phys 13(10):1479-1482

22. Spanos W Jr, Guse C, Perez C et al (1989) Phase II study of multiple daily fractionations in the palliation of advancedpelvic malignancies: preliminary report of RTOG 8502. Int J Radiat Oncol Biol Phys 17(3):659-661

23. Spanos W Jr, Perez CA, Marcus S et al (1993) Effect of rest interval on tumor and normal tissue response - a report of phase III study of accelerated split course palliative radiation for advanced pelvic malignancies. Int $\mathrm{J}$ Radiat Oncol Biol Phys 25:399-403

24. Spanos WJ Jr, Clery M, Perez CA et al (1994) Late effect of multiple daily fraction palliation schedules for advancedpelvic malignancies (RTOG 8502). Int J Radiat Oncol Biol Phys 29 (5):961-967

25. Spanos WJ Jr, Pajak TJ, Emami B et al (1996) Radiation palliation of cervical cancer. J Natl Cancer Inst Monogr 21:127-130

26. Yan J, Milosevic M, Fyles A, Manchul L et al (2011) A hypofractionated radiotherapy regimen (0-7-21) for advanced gynaecological cancer patients. Clin Oncol (R Coll Radiol) 23(7):476-481

27. Corn BW, Lanciano RM, Boente M et al (1994) Recurrent ovarian cancer. Cancer 74:2979-2983

28. Moore DH, Thomas GM, Montana GS et al (1998) Preoperative chemoradiation for advanced vulvar cancer: a phase II study of the Gynecologic Oncology Group. Int J Radiat Oncol Biol Phys 42:79-85

29. May LF, Belinson JL, Roland TA (1990) Palliative benefit of radiotherapy in advanced ovarian cancer. Gynecol Oncol 37:408-411

30. Tinger A, Waldron T, Peluso N et al (2001) Effective palliative radiotherapy in advanced and recurrent ovarian carcinoma. Int $\mathrm{J}$ Radiat Oncol Biol Phys 51:1256-1263 